\title{
Discovery of selective inhibitors of Glutaminase-2, which inhibit mTORC1, activate autophagy and inhibit proliferation in cancer cells
}

\author{
Yue-Zhi Lee ${ }^{1, *}$, Cheng-Wei Yang ${ }^{1, *}$, Hsin-Yu Chang ${ }^{1, *}$, Hsing-Yu Hsu ${ }^{1, *}$, Ih-Shen \\ Chen ${ }^{2}$, Hsun-Shuo Chang ${ }^{2}$, Chih-Hao Lee ${ }^{3}$, Jinq-Chyi Lee ${ }^{1}$, Chidambaram Ramesh \\ Kumar $^{1}$, Ya-Qi Qiu' ${ }^{1,4}$, Yu-Sheng Chao ${ }^{1}$ and Shiow-Ju Lee ${ }^{1}$ \\ ${ }^{1}$ Institute of Biotechnology and Pharmaceutical Research, National Health Research Institutes, Miaoli, Taiwan \\ 2 School of Pharmacy, College of Pharmacy, Kaohsiung Medical University, Kaohsiung, Taiwan \\ ${ }^{3}$ Department of Genetics and Complex Diseases, Division of Biological Sciences, Harvard School of Public Health, Boston, \\ Massachusetts, USA \\ ${ }^{4}$ Graduate Program of Biotechnology in Medicine, Institute of Molecular \& Cellular Biology, National Tsing Hua University, \\ Hsinchu, Taiwan \\ * These authors equally contributed to this work
}

Correspondence to: Shiow-Ju Lee, email: slee@nhri.org.tw

Keywords: AMPK; Autophagy; Glutaminase; mTOR; Raptor; ULK 1

Received: April 26, $2014 \quad$ Accepted: July 7, $2014 \quad$ Published: July 8, 2014

This is an open-access article distributed under the terms of the Creative Commons Attribution License, which permits unrestricted use, distribution, and reproduction in any medium, provided the original author and source are credited.

\section{ABSTRACT}

Glutaminase, which converts glutamine to glutamate, is involved in Warburg effect in cancer cells. Two human glutaminase genes have been identified, GLS (GLS1) and GLS2. Two alternative transcripts arise from each glutaminase gene: first, the kidney isoform (KGA) and glutaminase C (GAC) for GLS; and, second, the liver isoform (LGA) and glutaminase B (GAB) for GLS2. While GLS1 is considered as a cancer therapeutic target, the potential role of GLS2 in cancer remains unclear. Here, we discovered a series of alkyl benzoquinones that preferentially inhibit glutaminase $B$ isoform (GAB, GLS2) rather than the kidney isoform of glutaminase (KGA, GLS1). We identified amino acid residues in an allosteric binding pocket responsible for the selectivity. Treatment with the alkyl benzoquinones decreased intracellular glutaminase activity and glutamate levels. GLS2 inhibition by either alkyl benzoquinones or GLS2 siRNA reduced carcinoma cell proliferation and anchorageindependent colony formation, and induced autophagy via AMPK mediated mTORC1 inhibition. Our findings demonstrate amino acid sequences for selective inhibition of glutaminase isozymes and validate GLS2 as a potential anti-cancer target.

\section{INTRODUCTION}

Cancer cells tend to exhibit the Warburg effect, in which cellular metabolisms proceed via: 1) an altered glucose metabolism characterized by a high rate of glycolysis followed by lactic acid fermentation; and 2) up-regulated glutaminolysis by increasing glutaminase activity. Glutamine is essential for cancer cell proliferation, especially in the context of tricarboxylic acid (TCA) cycle anaplerosis. Glutaminase plays an essential role converting glutamine to glutamate, which is converted by glutamate dehydrogenase into $\alpha$-ketoglutarate. This $\alpha$-ketoglutarate enters the TCA cycle where it is involved in the production of ATP, nucleotides, certain amino acids, lipids, and glutathione in mitochondria [1]. Recently, glutaminase has emerged as a drug target for the development of glutaminase inhibitors for the treatment of cancerous disease [2]. However, only a few series of glutaminase inhibitors for GLS1 have been identified, and only partial in vivo anti-cancer efficacy has been demonstrated [3-6].

There are two human glutaminase genes, GLS (GLS1) and GLS2, located in chromosomes 2 and 12 respectively. Two alternative transcripts arise from each glutaminase gene: the kidney isoform (KGA) and 
glutaminase C (GAC) for $G L S 1$; and the liver isoform (LGA) and glutaminase B (GAB) for GLS2 [7].

The dimer-to-tetramer transition is essential for the enzymatic activation of glutaminase, which is active as a tetramer. Phosphate enhances this activation by facilitating the dimer-to-tetramer transition and substrate entry to the binding pocket by competing with the product glutamate $[3,8]$. Each glutaminase isoform has distinct kinetic and molecular characteristics, and the activity of each is also modulated by a variety of compounds, such as glutamate, citrate, calcium, certain long chain fatty acids, fatty acyl-CoA derivatives, TCA cycle intermediates, and protons [9], all of which are present under physiological conditions. Therefore, the kinetic and allosteric properties of the enzyme and the physiological environment are essential for regulating the enzyme activity in situ. Also, the differentiated amino acid residues or domains of the glutaminase isozymes provide potential binding pockets for selective allosteric modulators. For example, BPTES (bis-2-(5-phenylacetimido-1,2,4-thiadiazol-2-yl) ethyl sulfide), a GLS1 selective inhibitor, binds GAC through a differentiated gating loop close to the glutamine substrate binding site and locks the GAC tetramer into a nonproductive conformation [3].

In contrast to the disease indications of the KGA and GAC (GLS1) inhibitors described for anti-cancer [5, 6, 10], the biological role of GLS2 is still under exploration. GLS2 was found to regulate energy metabolism and antioxidant function as a tumor suppressor gene [1113]when ectopically overexpressed [11]; enrichment with LGA inhibits glioma cell growth and facilitates chemotherapeutic intervention [14]. However, knockdown of GLS2 expression sensitizes cervical cancer to ionizing radiation and thus reduces tumor size through decreasing cellular glutathione and NADH [15]. Therefore, more work is needed to clarify and demonstrate the role of GLS2 in cancer cell growth.

Glutaminase inhibition blocks the conversion of glutamine to glutamate and thus disables the conversion of glutamate into $\alpha$-ketoglutarate by glutamate dehydrogenase, which normally enters the TCA cycle to provide energy and bio-precursors for cancer cell growth. Autophagy is a catabolic process generally activated by such conditions of nutrient deprivation, and results in the autophagosomic-lysosomal degradation of bulk cytoplasmic contents. Autophagy is initiated and promoted by AMPK_ULK1 axis but inhibited by mTORC1 [16]. AMPK senses energy changes in cells and is activated when nutrients are depleted [17]. Rapamycin inhibits the Warburg effect $[18,19]$ and glutaminolysis feeds mTORC1 [20] so that a complex feedback exists between mTOR and glutaminase and Warburg effect. mTORC1 is also a critical regulator of autophagy induction and activation of mTORC1 suppresses autophagy. AMPK interacts with, phosphorylates, and activates ULK1 protein kinase, a key initiator of the autophagic process. Residue Ser317 of ULK1 is the main phosphorylation site for activation by AMPK [16]. In mammals, AMPK regulates autophagy, also involving inactivation of the mTORC1 pathway upon nutrient deficiency through two distinct pathways: phosphorylation for activation of Tuberin exchange factor or Raptor [21]. Conversely, mTOR phosphorylates ULK1 at Ser757 and disrupts the interaction between ULK1 and AMPK to inhibit autophagy [16]. In addition, the Beclin1 network also can induce and regulate autophagy via the formation of Beclin1-Vps34-Vps15 core complexes, and phagophore nucleation. The interaction of BCL2 with Beclin1 reduces autophagy. Phosphorylation of BCL2 by c-Jun N-terminal kinase 1 (JNK1) liberates Beclin1, allowing it to enter the nucleation process for autophagy [22]. Phosphorylation of Beclin1 by ULK1 at Ser15 (Ser14 in mice) is also required for full autophagic induction in mammals [23].

Natural products are a major source of inspiration in drug discovery [24, 25], and constitute a good resource for those seeking novel glutaminase inhibitors. While GLS1 is emerging as a therapeutic target for anticancer drugs $[5,6,10]$, the biological role of GLS2 is still under exploration. Herein, we disclose a series of natural alkyl benzoquinones with a glutaminase inhibitory effect. Through homologous modeling and mutagenesis, the alkyl benzoquinone binding site was simulated and demonstrated to be an allosteric pocket. From the allosteric pocket, two divergently differentiated residues were found to account for the selective inhibition for GAB (an isoform of GLS2) over KGA (an isoform of GLS1). Furthermore, inhibition of glutaminase activity by the active alkyl benzoquinone AV-1 in carcinoma cells led to autophagy via AMPK-mediated ULK1 activation and mTORC1 inhibition, ultimately leading to inhibition of cancer cell growth.

\section{RESULTS}

\section{Purification of the recombinant human KGA and GAB for screening glutaminase inhibitors and analysis of structure-activity relations and inhibition modes}

A collection of $\sim 200$ natural products isolated from a variety of indigenous Taiwanese plants were submitted for screening against KGA. Human recombinant KGA and GAB (Figure 1A) from E. coli were cloned, expressed and purified. Characterization of its enzymatic properties showed that the recombinant GAB but not KGA responded to its substrate glutamine in allosteric and positive cooperative manners (Figure 1B and 1C), as previously reported for these enzymes purified from human biopsies [26]. We first screened the compound collection described above for inhibitory activity against the recombinant 
Table 1: Natural alkyl benzoquinones inhibit the enzymatic activities of human KGA and GAB. See Materials and Methods for the measurement of the $\mathrm{IC}_{50}$ values. Shown structures are alkyl benzoquinones and alkyl phenols isolated from plants Ardisia virens (AV series) and A. kusukuensis (AK series)[27, 28]

\section{AV series}



$\underbrace{R_{R_{2}}^{R_{1}}}_{\mathbf{R}_{3}}$

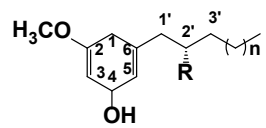

$\mathrm{H}_{3} \mathrm{CO} \underbrace{\mathrm{OCOCH}_{1}^{2} H_{3}^{2}}_{\mathrm{OH}}$
AV-1: $R_{1}=\mathrm{OCOCH}_{3}, \mathrm{R}_{2}=\mathrm{H}, \mathrm{n}=11$

AV-2: $\mathrm{R}_{\mathbf{1}}=\mathrm{OCOCH}_{3}, \mathrm{R}_{2}=\mathrm{H}, \mathrm{n}=\mathbf{9}$

AV-3: $\mathrm{R}_{1}=\mathrm{OCOCH}_{3}, \mathrm{R}_{2}=\mathrm{OH}, \mathrm{n}=11$

AV-4: $\mathrm{R}_{1}=\mathrm{OCOCH}_{3}, \mathrm{R}_{2}=\mathrm{OH}, \mathrm{n}=9$

AV-5: $R_{1}=R_{2}=H, n=9$

AV-6: $R_{1}=R_{2}=H, n=11$

AV-7: $R_{1}=H, R_{2}=O H, n=9$

AV-8: $R_{1}=\mathrm{OCOCH}_{3}, \mathrm{R}_{2}=\mathrm{H}, \mathrm{R}_{3}=\mathrm{OH}, \mathrm{R}_{4}=\mathrm{OCH}_{3}, \mathbf{n = 9}$

AV-9: $R_{1}=R_{3}=\mathrm{OCOCH}_{3}, R_{2}=H, R_{4}=O_{3} H_{3}, n=9$

AV-10: $R_{1}=\mathrm{OCOCH}_{3}, R_{2}=\mathrm{CHO}, \mathrm{R}_{3}=\mathrm{OH}, \mathrm{R}_{4}=\mathrm{OCH}_{3}, \mathrm{n}=9$

AV-11: $R_{1}=R_{3}=O \operatorname{OCCH}_{3}, R_{2}=H, R_{4}=O_{3}, n=11$

AV-12: $\mathrm{R}=\mathrm{OH}, \mathrm{n}=11$

AV-13: $R=H, n=3$

AV-14

\section{AK series}

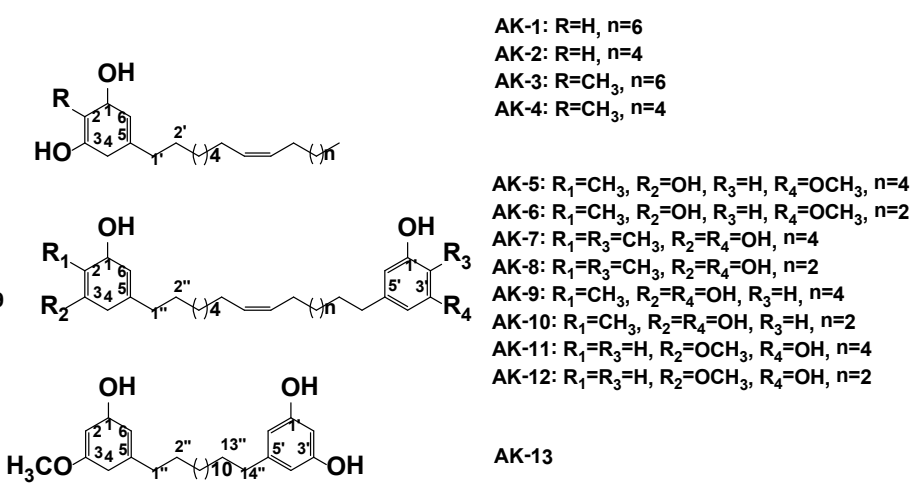

\begin{tabular}{|c|c|c|}
\hline & \multicolumn{2}{|c|}{ Glutaminase } \\
\hline & KGA & GAB \\
\hline Compound ID & $\mathbf{I C}_{50}(\mu \mathbf{M})$ & $\mathbf{I C}_{50}(\mu \mathrm{M})$ \\
\hline $\mathrm{AV}-1$ & $2.1 \pm 0.1$ & $0.28 \pm 0.02$ \\
\hline$A V-2$ & $3.9 \pm 0.4$ & $0.29 \pm 0.05$ \\
\hline$A V-3$ & $>50$ & $25.1 \pm 4.5$ \\
\hline$A V-4$ & $>50$ & $>50$ \\
\hline$A V-5$ & $>50$ & $32.5 \pm 2.7$ \\
\hline$A V-6$ & $>\mathbf{5 0}$ & $>\mathbf{5 0}$ \\
\hline $\mathrm{AV}-7$ & $>50$ & $>50$ \\
\hline$A V-8$ & $2.9 \pm 0.1$ & $0.26 \pm 0.05$ \\
\hline AV-9 & $>\mathbf{5 0}$ & $31.7 \pm 6.7$ \\
\hline$A V-10$ & $>\mathbf{5 0}$ & $23.1 \pm 4.9$ \\
\hline AV-11 & $69.0 \pm 3.1$ & $5.7 \pm 1.1$ \\
\hline AV-12 & $52.4 \pm 15.5$ & $7.6 \pm 2.0$ \\
\hline AV-13 & $52.7 \pm 2.7$ & $>\mathbf{5 0}$ \\
\hline AV-14 & $41.0 \pm 5.2$ & $4.7 \pm 1.3$ \\
\hline AK-1 & $73.0 \pm 3.0$ & $2.9 \pm 0.6$ \\
\hline AK-2 & $76.5 \pm 0.9$ & $2.8 \pm 0.3$ \\
\hline AK-3 & $76.9 \pm 0.8$ & $2.7 \pm 10.4$ \\
\hline AK-4 & $59.8 \pm 4.6$ & $>50$ \\
\hline AK-5 & $70.0 \pm 1.6$ & $2.3 \pm 0.4$ \\
\hline AK-6 & $36.7 \pm 3.2$ & $2.5 \pm 0.3$ \\
\hline AK-7 & $17.5 \pm 2.6$ & $2.9 \pm 0.8$ \\
\hline AK-8 & $13.9 \pm 1.5$ & $3.1 \pm 0.5$ \\
\hline AK-9 & $11.9 \pm 1.2$ & $2.3 \pm 0.5$ \\
\hline AK-10 & $11.7 \pm 1.3$ & $2.1 \pm 0.1$ \\
\hline AK-11 & $13.4 \pm 2.2$ & $2.9 \pm 0.5$ \\
\hline AK-12 & $9.6 \pm 1.2$ & $2.9 \pm 0.6$ \\
\hline AK-13 & $14.4 \pm 2.3$ & $2.8 \pm 0.4$ \\
\hline DON & $59.9 \pm 8.8$ & ND* \\
\hline BPTES & $0.08 \pm 0.01$ & $63.6 \pm 3.8$ \\
\hline ND: not determined & & \\
\hline
\end{tabular}


A

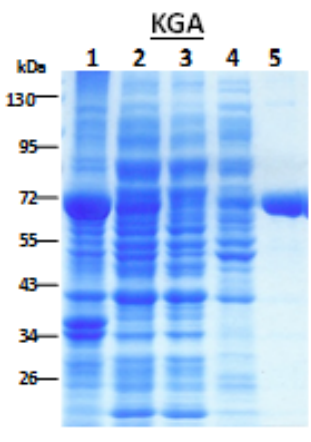

GAB



D

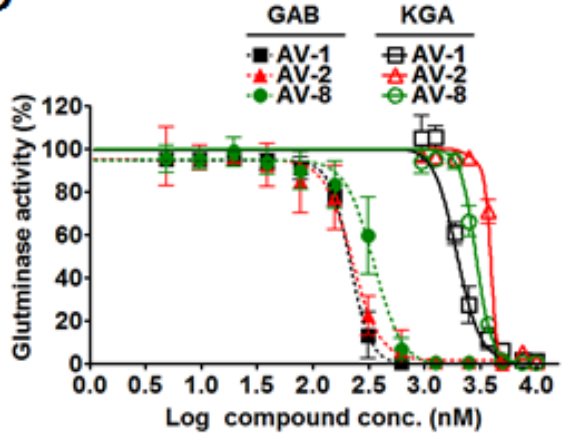

B KGA
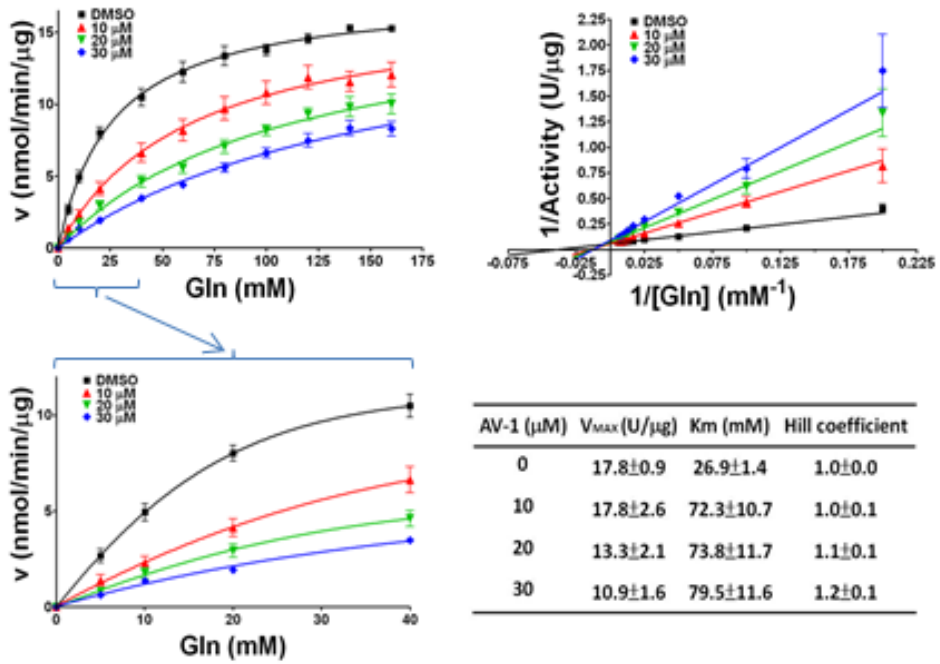

\begin{tabular}{cccc}
\hline AV-1 $(\mu \mathrm{M})$ & $V_{\max }(\mathrm{U} / \mu \mathrm{g})$ & $\mathrm{Km}(\mathrm{mM})$ & Hill coefficient \\
\hline 0 & $17.8 \pm 0.9$ & $26.9 \pm 1.4$ & $1.0 \pm 0.0$ \\
10 & $17.8 \pm 2.6$ & $72.3 \pm 10.7$ & $1.0 \pm 0.1$ \\
20 & $13.3 \pm 2.1$ & $73.8 \pm 11.7$ & $1.1 \pm 0.1$ \\
30 & $10.9 \pm 1.6$ & $79.5 \pm 11.6$ & $1.2 \pm 0.1$ \\
\hline
\end{tabular}

\section{C}


\begin{tabular}{cccc}
\hline AV-1 $(\mu \mathrm{M})$ & $V_{\max }(\mathrm{U} / \mu \mathrm{gg})$ & $\mathrm{Km}(\mathrm{mM})$ & Hill coefficient \\
\hline 0 & $20.2 \pm 0.7$ & $19.2 \pm 0.7$ & $1.4 \pm 0.1$ \\
0.2 & $21.5 \pm 2.0$ & $28.2 \pm 0.3$ & $1.4 \pm 0.1$ \\
0.4 & $17.4 \pm 2.0$ & $26.6 \pm 0.9$ & $1.4 \pm 0.1$ \\
0.6 & $11.0 \pm 1.5$ & $30.6 \pm 2.7$ & $1.3 \pm 0.1$ \\
\hline
\end{tabular}

Figure 1: Kinetic studies on the inhibition of alkyl benzoquinones in glutaminases. (A) Escherichia coli BL21(DE3)pLysS harboring pET28a(+)-KGA or $\mathrm{pET} 28 \mathrm{a}(+)-\mathrm{GAB}$ was propagated in Luria-Bertani broth medium in the presence of kanamycin, from which KGA or GAB protein expression was induced by $0.5 \mathrm{mM}$ IPTG. The resultant cell lysate was sonicated and centrifugated for fractions of cell pellet (lane 1) and supernatant (lane 2). The supernatant was passed through an affinity column (HisTrapTM HP) and the flowthrough was shown in lane 3, as well as the washes with $100 \mathrm{mM}$ and $150 \mathrm{mM}$ of imidazole for KGA(left panel) or $50 \mathrm{mM}$ of imidazole for GAB(right panel) were shown in lane 4. Lane 5 shown was the eluent with $500 \mathrm{mM}$ of imidazole for KGA(left panel) or $200 \mathrm{mM}$ of imidazole for GAB(right panel). Molecular markers were indicated as kDa. (B) Inhibition mode of active alkyl benzoquinone AV-1 for KGA in the absence of phosphate. (C) Inhibition mode of active alkyl benzoquinone AV-1 for GAB in the absence of phosphate. The Cheng-Prusoff equation, $\mathrm{Ki}=\mathrm{IC}_{50} /\left(1+[\mathrm{S}] / \mathrm{K}_{\mathrm{m}}\right)$, was used to calculate $\mathrm{Ki}$ values of $\mathrm{AV}-1$ for $\mathrm{KGA}=1.20 \pm 0.06 \mu \mathrm{M}$ and $\mathrm{GAB}=0.14 \pm 0.01 \mu \mathrm{M}$.

(D) Dose dependent inhibition of alkyl benzoquinones against the purified recombinant KGA and GAB in the absence of phosphate. 
$\mathrm{KGA}$, and then determined the $\mathrm{IC}_{50}$ values against KGA and GAB for the potent compounds (Table 1, Figure 1D).

Among the active hit compounds, a series of alkyl benzoquinones from Ardisia. virens (Table 1, AV series) were found to exhibit inhibition of human KGA and GAB (Table $1 \&$ Figure 1D). The keto (hydroxyl) groups at positions 1 and 4 on the benzoquinone core and the acetate group at position 2' were all found to be essential for potency for either KGA or GAB inhibition, by comparing the most potent compounds AV-1, AV-2, and AV-8 (KGA, $\mathrm{IC}_{50}$ values $2.1 \pm 0.1 \mu \mathrm{M} ; 3.9 \pm 0.4 \mu \mathrm{M} ; 2.9 \pm 0.1 \mu \mathrm{M} ; \mathrm{GAB}$, $\mathrm{IC}_{50}$ values $0.28 \pm 0.02 \mu \mathrm{M} ; 0.29 \pm 0.05 \mu \mathrm{M} ; 0.26 \pm 0.05 \mu \mathrm{M}$ respectively) to the other $\mathrm{AV}$ compounds $\left(\mathrm{KGA}, \mathrm{IC}_{50}\right.$ values $>40 \mu \mathrm{M}$; GAB, $\mathrm{IC}_{50}$ values $\left.>4.7 \mu \mathrm{M}\right)($ Table 1$)$.

As the AV compounds were also found to be selective for GAB over KGA, the inhibition mode of AV-1 (the most active) was investigated. AV-1 inhibited both KGA and GAB in a mixed non-competitive inhibition; when AV-1 concentration was increased from 0 to $30 \mu \mathrm{M}$, the $\mathrm{K}_{\mathrm{m}}$ values increased by 2.9- and 1.6-fold and the $\mathrm{V}_{\max }$ values decreased to $61 \%$ and $54 \%$ for $\mathrm{KGA}$ and $\mathrm{GAB}$ respectively (Figure $1 \mathrm{~B} \& 1 \mathrm{C}$ ). AV-1 exhibited Ki values of $1.20 \pm 0.06 \mu \mathrm{M}$ for KGA and $0.14 \pm 0.01 \mu \mathrm{M}$ for $\mathrm{GAB}$, as calculated using the Cheng-Prusoff equation; the AV-1 exerted a positive cooperative manner with the substrate glutamine in inhibiting GAB with a Hill coefficient of 1.3-1.4 and an independent manner in inhibiting KGA with a Hill coefficient of $\sim 1.0-1.2$ (Figure 1B \& 1C). In conclusion, the mechanisms by which AV-1 inhibits GAB and KGA are different.

\section{Homologous modeling, docking studies, and binding site analysis}

Based on the structure-activity relationships and inhibition modes analyzed above, we sought to determine the possible location of the AV-1-binding site on GAB [3]. Homologous modeling for GAB was conducted using the human GLS1 crystal structure 3UO9 and AutoDock Vina (http://vina.scripps.edu/). AutoDock Vina was further used to carry out a docking study for compound AV-1 over the entire surface of the homologous modeled GAB dimer or monoer. The simulated pocket for AV-1 binding from docking showed major hydrogen bond and van der Waals interactions between $\mathrm{AV}-1$ with the GAB residues H408, Q452, K453, S456, H461 and H472 (Figure 2A). The binding pocket is located at the C-terminal end of the GAB monomer, close to the dimer interaction interface. However, no inter-monomeric interactions were found for $\mathrm{AV}-1$ and therefore it is assumed that one AV- 1 binds to one GAB monomer as shown in Figure 2B. Further sequence comparison of $\mathrm{KGA}$ and $\mathrm{GAB}$ at the $\mathrm{AV}-1$ binding region indicate that the $\mathrm{GAB}$ residues Q452 and $\mathrm{K} 453$ were differentiated from those corresponding residues of KGA H519 and D520 (Figure 2C).
Mutagenesis studies to validate the interactions for selectivity were next carried out. When the residues Q452 and $\mathrm{K} 453$ in GAB were mutated into His or Asp to give the mutants Q452H, K453D or Q452H/K453D, the inhibitory effect significantly decreased: $\mathrm{IC}_{50}$ values of $0.28 \pm 0.02$ $\mu \mathrm{M}$ (wild type), $1.33 \pm 0.32 \mu \mathrm{M}(\mathrm{Q} 452 \mathrm{H}), 4.17 \pm 0.11$ $\mu \mathrm{M}(\mathrm{K} 453 \mathrm{D})$, and $5.51 \pm 0.28 \mu \mathrm{M}(\mathrm{Q} 452 \mathrm{H} / \mathrm{K} 453 \mathrm{D})$ were measured. These results demonstrate that the effects of the two GAB point mutations in decreasing AV-1 potency were additive. On the contrary, when the corresponding residues H519 and D520 in KGA were mutated into Gln or Lys to give the mutants H519Q, D520K or H519Q/ D520K, the inhibitory effect increased: $\mathrm{IC}_{50}$ values of 2.1 $\pm 0.1 \mu \mathrm{M}$ (wild type), $1.67 \pm 0.12 \mu \mathrm{M}$ (H519Q), $0.29 \pm$ $0.03 \mu \mathrm{M}(\mathrm{D} 520 \mathrm{~K})$, and $0.23 \pm 0.03 \mu \mathrm{M}(\mathrm{H} 519 \mathrm{Q} / \mathrm{D} 520 \mathrm{~K})$ were measured. These results demonstrate that the effects of the two KGA point mutations reversely increased AV-1 potency.

Moreover, AV-1 comparably inhibited the wild type and the two KGA mutants corresponding to those of GAC (F318Y/F322S; Y394L) (Figure 2D) while these two KGA mutant enzymes were resistant to BPTES inhibition due to loss of key interactions demonstrated from cocrystallization data previously reported (Figure 2D) [3]. Therefore, we conclude that the interactions between $\mathrm{AV}-1$ and $\mathrm{GAB}$ residues $\mathrm{Q} 452$ and $\mathrm{K} 453$ determine the selectivity for GAB over KGA.

\section{GLS2 inhibition via AV-1 compound or RNA silencing exhibits anti-cancer cell proliferation and anti- anchorage-independent colony formation}

$\mathrm{GAB}$ and KGA protein expression levels were found to be higher in human hepatoma HepG2 and lung carcinoma A549 cells than those in fibroblast cell lines Detroit 551 and WI-38, whilst GAC expression levels were not significantly changed (Figure 3A). LGA protein expression was low in each cell line but expressed slightly more in HepG2. KGA exhibited two major forms of protein, one of which was multi-phosphorylated as demonstrated by an addition of phosphatase experiment that affected the phosphorylation of KGA and changed the electrophoretic mobility of KGA protein in SDS gel (Figure 3A \& Figure S1). The capacity of these compounds to inhibit growth of the cell lines A549, HepG2, MCF7 (human breast carcinoma), NCI-H460 (human lung carcinoma), and SF268 (human glioblastoma) (Figure 3B, Table S1, and previously described [27, 28]) were found to be consistent with their glutaminase inhibition (Table 1). Moreover, AV-1 inhibited colony formation of HepG2 cells, as did GLS1 inhibitor BPTES, a reference control (Figure 3C, left panel). After further knocking down GLS2 protein expression using a silencing RNA approach (see Materials and Methods), the A549 and HepG2 cell 
A



B
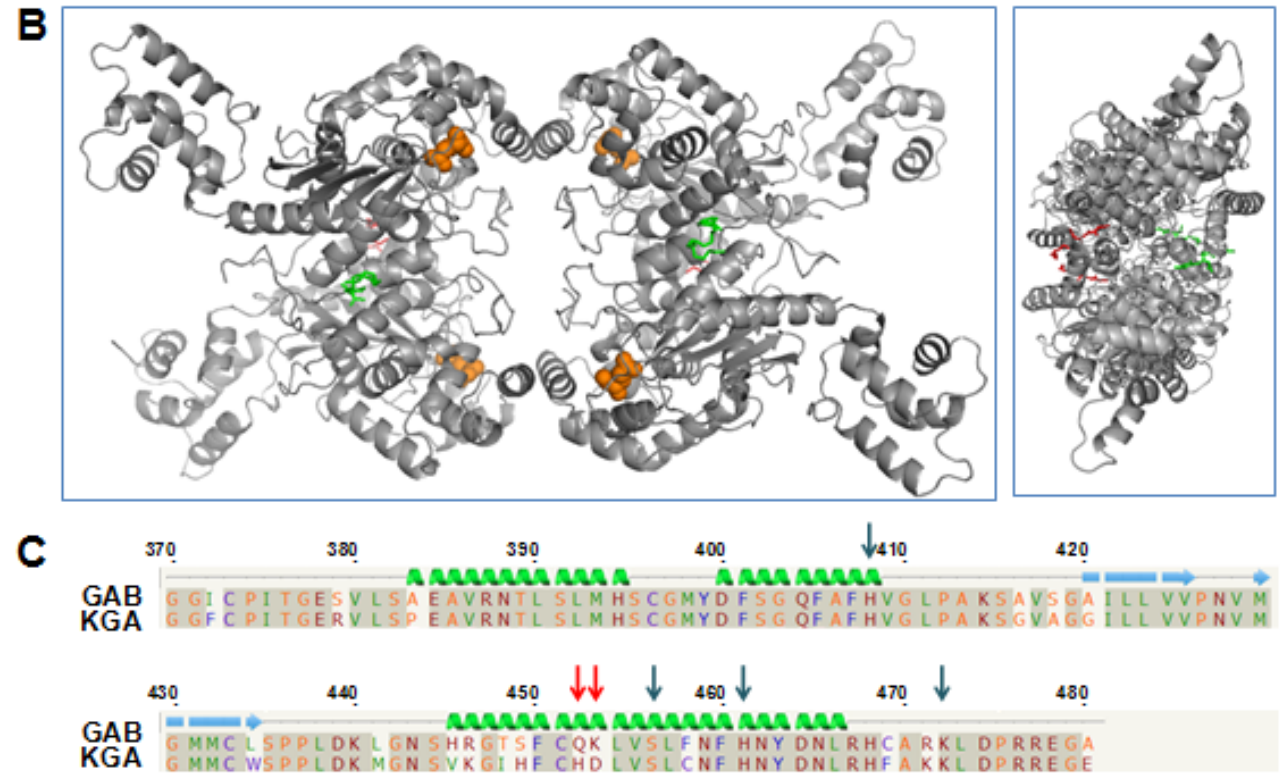

\begin{tabular}{|c|c|c|c|c|}
\hline Compound & Glutaminase & $\begin{array}{l}\mathrm{IC}_{50} \\
(\mu \mathrm{M}) \\
\end{array}$ & $\begin{array}{l}\text { Fold change } \\
\text { in } I_{50} \text { value }\end{array}$ & $\begin{array}{l}\text { Selectivity Index } \\
\text { for GAB over KGA }\end{array}$ \\
\hline \multirow[t]{5}{*}{ AV-1 } & GAB & & & \\
\hline & WT & $0.28 \pm 0.02$ & 1.0 & 7.50 \\
\hline & Q452H & $1.33 \pm 0.32$ & 4.7 & 0.63 \\
\hline & K453D & $4.17 \pm 0.11$ & 14.9 & 0.50 \\
\hline & Q452HVK453D & $5.15 \pm 0.28$ & 18.4 & 0.41 \\
\hline \multirow[t]{7}{*}{ AV1 } & KGA & & & \\
\hline & WT & $2.10 \pm 0.10$ & 1.0 & $\mathbf{N A}^{*}$ \\
\hline & H519Q & $1.67 \pm 0.12$ & 0.79 & NA \\
\hline & D520K & $0.29 \pm 0.03$ & 0.14 & NA \\
\hline & H519CDD520K & $0.23 \pm 0.03$ & 0.11 & NA \\
\hline & F318Y/F322S & $2.40 \pm 0.00$ & 1.1 & NA \\
\hline & Y394L & $1.40 \pm 0.20$ & 0.67 & NA \\
\hline \multirow[t]{4}{*}{ BPTES } & KGA & & & \\
\hline & $\overline{\text { WT }}$ & $0.080 \pm 0.001$ & 1.0 & NA \\
\hline & F318Y/F322S & $>100$ & $>1250$ & NA \\
\hline & Y394L & $>100$ & $>1250$ & NA \\
\hline
\end{tabular}

*NA: not avaikable.

Figure 2: Docking studies and binding site analyses of AV-1 in GAB. (A) Docking simulation of AV-1 binding site in GAB. Magenta: H408 histidine; Yellow: Q452 glutamine; Orange: K453 lysine; Red: S456 serine; Cyan: H461 histidine; Blue: K472 lysine; Dashed lines: hydrogen bond interactions. AV-1 chemical structure was shown with interacted residues of GAB. (B) Overview of the proposed association of AV-1 in GAB tetramer. Left panel presents a top view and right panel a side view. AV-1 is presented in red and green stick figure for clarity, and the glutamate molecules are shown as orange spheres. (C) Primary sequence alignment of human KGA and human GAB around the simulated AV-1 binding site. Key interactive residues are denoted with arrows. Mutation sites for selectivity of GAB over KGA are denoted with red arrows. (D) $\mathrm{IC}_{50}$ values and selectivity index of AV-1 \& BPTES for GAB or KGA wild-type and mutant enzymes. Mutations at residues Q452 and K453 of GAB into corresponding residues of KGA created GAB mutants GAB_Q452H, GAB_K453D, and GAB_Q452H/K453D. Mutations at residues H519 and D520 of KGA into corresponding residues of GAB created KGA mutants KGA_H519Q, KGA_D520K, and KGA_H519Q/D520K. Mutations at residues F318/F322 and Y394 of KGA into corresponding residues of GAB or bacterial glutaminase created KGA mutants KGA_F318Y/F322S and KGA_Y394L [3]. 
A



B

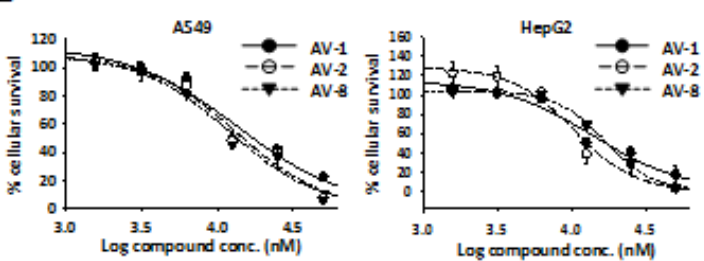

$\mathrm{C}$

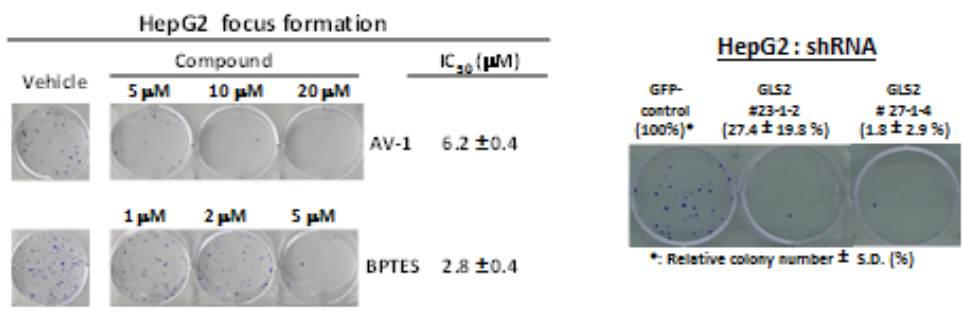

D
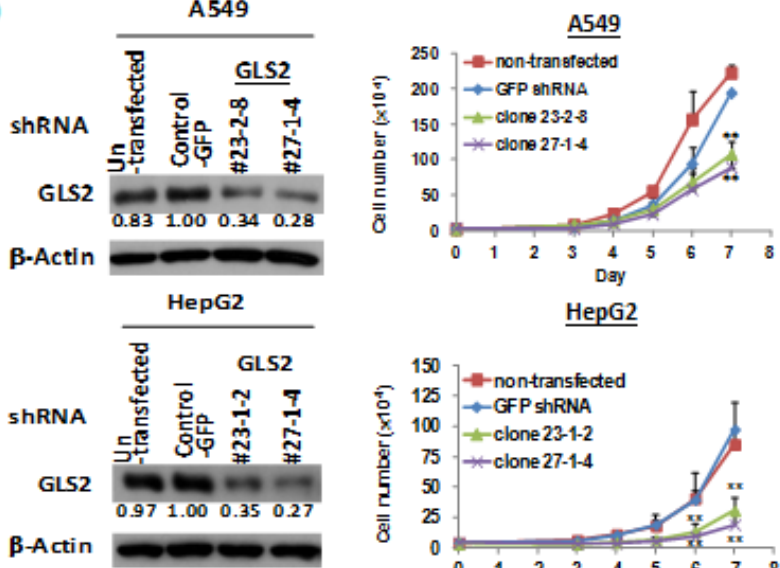

E


A549

$\mathrm{F}$
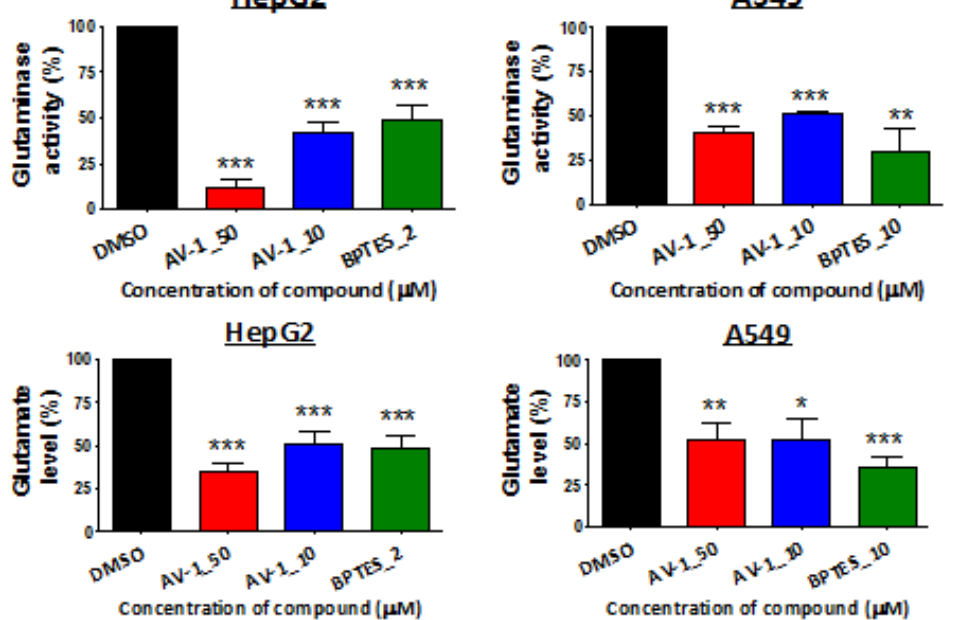

A549

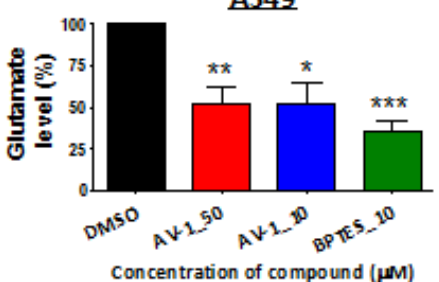

Figure 3: GLS2 inhibition via AV-1 compound or RNA silencing exhibits anti-cancer cell proliferation and antianchorage-independent colony formation. (A) GLS1 and GLS2 protein expressions in carcinoma cells HepG2 and A549 as well as normal fibroblast cell lines Detroit 551 and WI-38. (B) Dose dependent growth inhibition of active alkyl benzoquinones against carcinoma cells HepG2 and A549. (C) The effect of GLS2 inhibition via AV-1 or GLS2 knockdown on the colony formation of HepG2 cells. (D) Knockdown GLS2 expression significantly inhibited the carcinoma HepG2 and A549 cells growth. Shown are western blot analysis for GLS2 expression levels and growth curves of un-transfected (parental) cells, shRNA-control cell (shRNA-control) and GLS2 shRNA knockdown cells. ${ }^{* *} \mathrm{P}<0.005$. (E) AV-1 treatments decreased intracellular glutaminase activity. (F) AV-1 treatments decreased intracellular glutamate levels. The cells lysates treated with AV-1 or BPTES were subject to glutaminase activity assay and glutamates levels (see experimental procedures). * $\mathrm{P}<0.05,{ }^{* *} \mathrm{P}<0.01,{ }^{* * *} \mathrm{P}<0.001$. See also Figure $\mathrm{S} 1$ and Table $\mathrm{S} 1$. 
growth were decreased by $\sim 45-81 \%$ (Figure 3D) and colony formation inhibited accordingly (Figure 3C, right panel). AV-1 treatment significantly decreased intracellular glutaminase activity and product glutamate levels (Figure 3E \& 3F). Therefore, the alkyl benzoquinones inhibited carcinoma cell growth by inhibiting cellular glutaminase activity.

\section{AV-1 compound induces autophagy and activates AMPK}

Anti-cancer agents usually induce diversified forms of cell death including apoptosis and autophagy [29]. To examine whether AV-1 inhibited cell growth leads to cell death, the cell death-related apoptosis and autophagy pathways were examined in AV-1 treated HepG2 cells. Autophagic activity was monitored by tracking the conversion of LC3-I to LC3-II. A reliable marker of autophagosome LC3 is a mammalian homolog of yeast Atg8 [30]. It was found that LC3-II production was induced after the treatment of $\mathrm{HepG} 2$ cells with $\mathrm{AV}-1$ for 24-72 h (Figure 4A), but the apoptosis marker-cleaved caspase 3 [31] was not detected (Figure 4A). Because glutaminase inhibition in cancer cells decreases cellular energy production and results in nutrient depletion [5] (Figures 3 B-F); and because autophagy is generally activated by conditions of nutrient deprivation [16], we concluded that induction of autophagy by AV-1 treatment was responsible for cell death (Figures 3B-E and 4A).

Autophagy is initiated and promoted by activation of AMPK_ULK1 axis signaling, but inhibited by mTORC1 [16]. Therefore, we examined the effects of AV-1 treatment on these signaling pathways in HepG2 carcinoma cells. Our investigation of the autophagy-related AMPK signaling pathway regulated by AV-1 showed: i) AMPK was phosphorylated for activation $0.5-3 \mathrm{~h}$ after AV-1 treatment (Fig 4B); ii) the moderate phosphorylation of ULK1 at Ser317 by AMPK [16] occurred at 3-12 $\mathrm{h}$ while the phosphorylation of ULK1 at Ser757 by mTOR [16] was significantly decreased up to $72 \mathrm{~h}$ after AV-1 treatment (Fig 4C); iii) The protein and phosphorylation (at Ser15) levels of autophagic Beclin1 were not significantly affected and the level of phosphorylated BCL2 decreased up to $72 \mathrm{~h}$ after AV-1 treatment (Figure 4C). These results indicate that the AMPK and ULK1 were activated while mTORC1 activity was suppressed in HepG2 cells treated with AV-1. Moreover, AV-1 induced autophagy is independent of Beclin1 and phosphorylated BCL2 signaling.

\section{AV-1 compound treatment results in mTORC1 inhibition through activated AMPK and inhibited P13K/Akt}

To investigate how mTORC1 activity is regulated by AV-1, phosphorylation of the mTORC1 components mTOR, Raptor, and Tuberin were separately examined (Fig. 5A). Firstly, phosphorylation at Ser2448 and autophosphorylation at Ser2481 of mTOR for activation via PI3K/AKT $[32,33]$ started to level off around $12 \mathrm{~h}$, and then gradually increased until phosphorylation was complete at $72 \mathrm{~h}$ in AV-1 treated HepG2 cells. On the contrary, gradually decreasing levels of phosphorylation for these two sites occurred in the DMSO vehicle
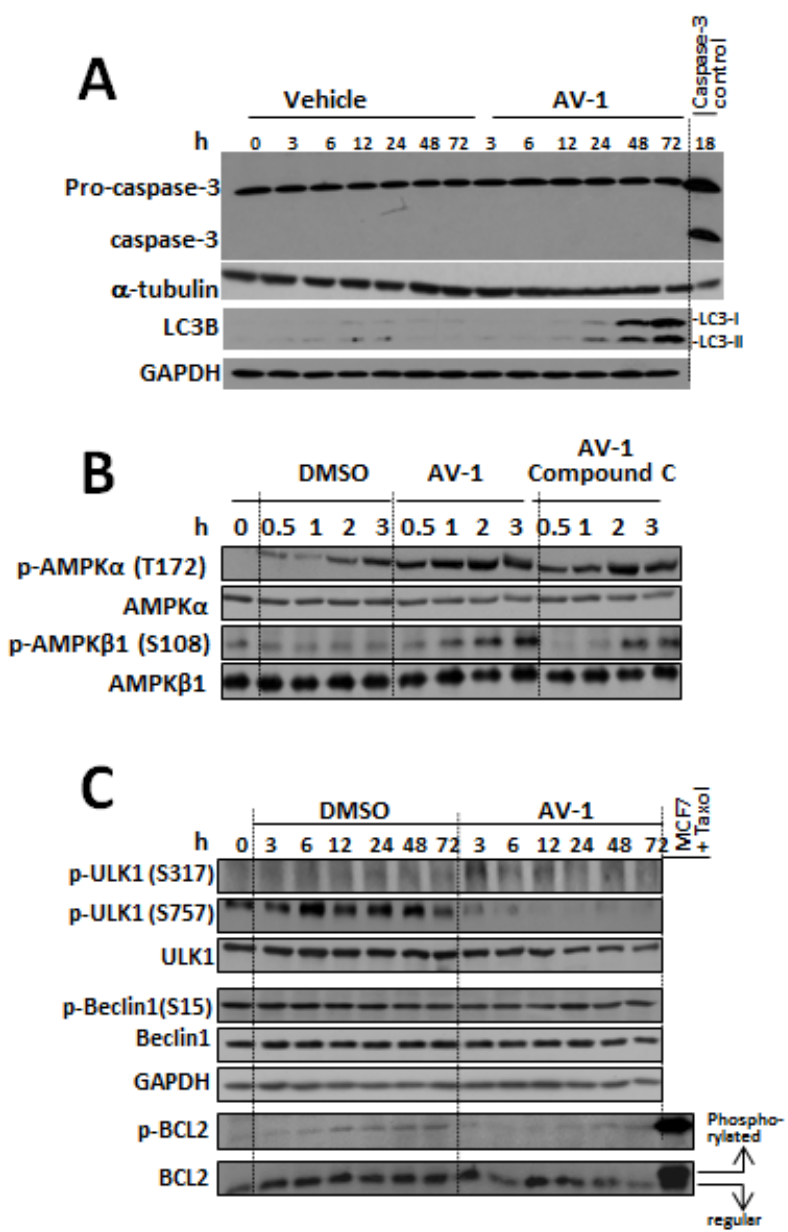

Figure 4: AV-1 induced cell autophagy through activated AMPK and ULK1. (A) Effects of AV-1 treatment on the induction of apoptosis and autophagy in HepG2 cells manifested by cleavage caspase 3 or LC3B. (B) The effects of AV-1 on AMPK activation. C. The effects of AV-1 on activation of ULK1 and BCL2 associated Beclin1. HepG2 cells were treated with vehicle (DMSO) or AV-1 for various times as indicated prior to cell lysis for western blot analysis with the antibodies indicated. The results shown are representative of three independent experiments. 
treatment.

Secondly, another nutrient sensing residue, Thr2446 of mTOR, which undergoes phosphorylation by AMPK to give inactivated mTOR under conditions of nutrient deprivation [34], underwent significant phosphorylation in the first $24 \mathrm{~h}$, only to become dephosphorylated around 48-72 h in HepG2 cells treated with AV-1. On the contrary, HepG2 cells treated with DMSO vehicle did not show phosphorylation at Thr2446 of mTOR in the first $12 \mathrm{~h}$, but a gradual increase in phosphorylation occurred from $24 \mathrm{~h}$ up to $72 \mathrm{~h}$ (Figure 5A). These results suggest that mTOR activity was inhibited for the first $12 \mathrm{~h}$ after AV-1 treatment, after which it became activated again.

Thirdly, residue Thr1462 of Tuberin (an AKT phosphorylation site to activate mTORC1) [35] was phosphorylated all over the 72 h-time course and even getting highly phosphorylated at $48-72 \mathrm{~h}$ period in the HepG2 cells treated with DMSO. However, AV-1 treatment disabled Tuberin phosphorylation at Thr1462 over the same time period (Figure $5 \mathrm{~A}$ ). These results suggested that AV-1 treatment likely inhibited PI3K/AKT activity and the resultant Tuberin (dephosphorylated at Thr1462) did contribute to inhibition of the mTORC1 activity.
Fourthly, a dramatic increase in phosphorylation of Raptor at Ser792 (a site for AMPK direct phosphorylation) for mTORC1 inactivation upon AV-1 treatment was observed compared to DMSO vehicle controls (Figure 5A upper panel).

Fifthly, mTORC1 inhibition was further manifested by the elimination of phosphorylation of p70S6K and 4E-BP, further downstream targets of mTORC1 by AV-1 treatment (Figure 5A lower panel).

Lastly, compound C (an AMPK inhibitor) attenuated the AV-1 induced phosphorylation of Raptor at Ser792 and Tuberin at Ser1387 (Figure 5B). In response to nutrient deprivation, Ser1387 of Tuberin gets phosphorylated by AMPK which also leads to mTORC1 inactivation [36]. On the contrary, compound $\mathrm{C}$ reversed the decreased phosphorylation of ULK1 Ser757 (Figure 5B and Figure S2).

Thus, we conclude that AV-1 inhibited intracellular glutaminase activity and resulted in decreased glutamate level in HepG2 cells, leading to autophagy by AMPK activation. AV-1 treatment activated AMPK which moderately phosphorylated ULK1 at Ser317 for autophagy initiation, and strongly phosphorylated Raptor at Ser792 and Tuberin at Ser1387 for mTORC1 inactivation for
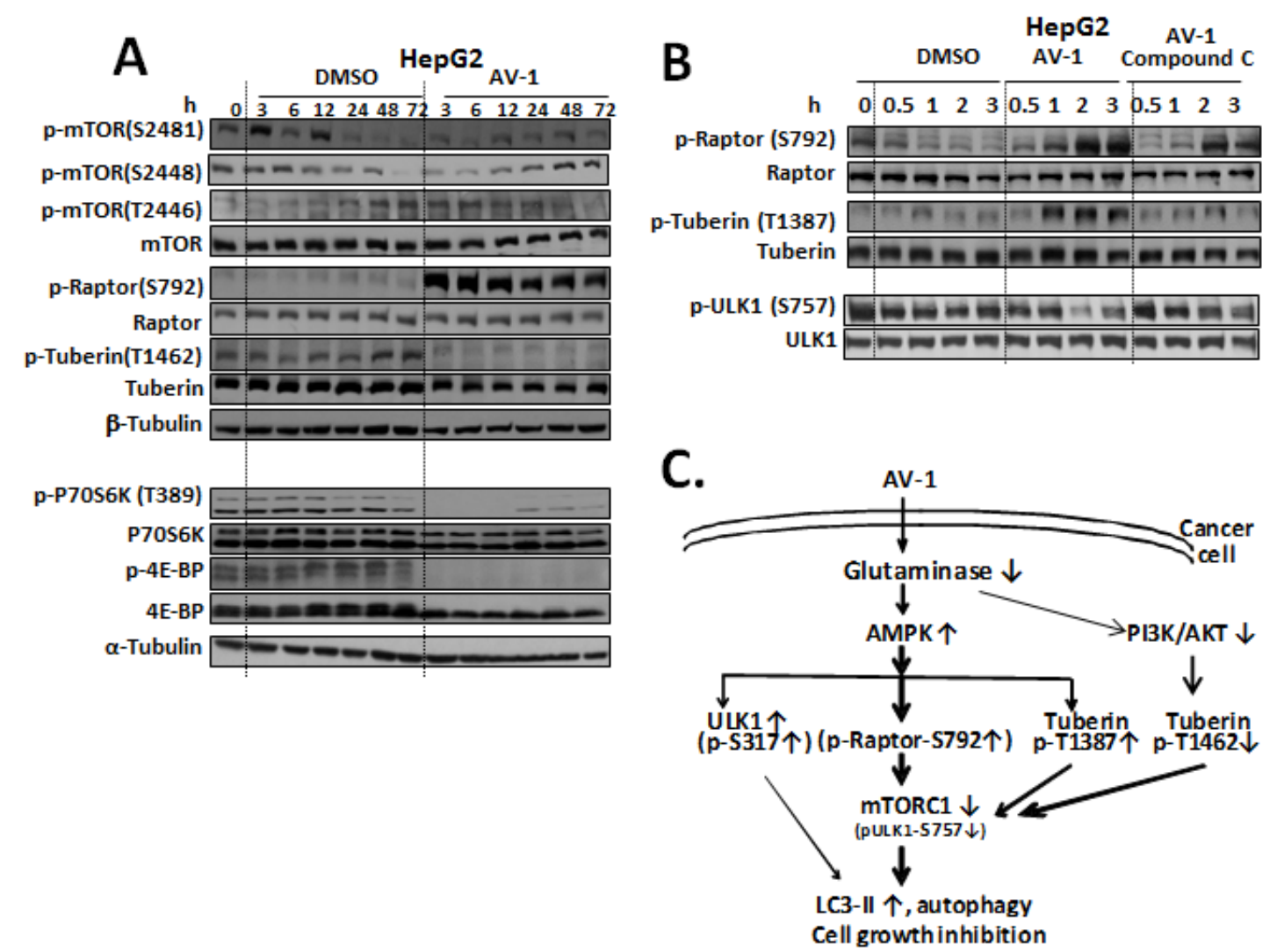

Figure 5: AV-1 inhibits mTORC1 through AMPK activation. (A) Effects of AV-1 on mTORC1 inactivation. (B) AMPK inhibition reverses the AV-1 associated mTORC1 inhibition. HepG2 cells were treated with vehicle (DMSO), AV-1, or Compound C (an inhibitor of AMPK) for various times as indicated prior to cell lysis for western analysis with the antibodies indicated. (C) A scheme illustrating that AV-1 inhibits glutaminase, activates autophagic pathway through AMPK activation and mTORC1 inhibition. The results shown are representative of three independent experiments. See also Figure S2. 
ultimate autophagy. Figure $5 \mathrm{C}$ illustrated the effects of AV-1 in cell growth inhibition and induction of autophagy through AMPK mediated moderate ULK1 activation and profound $\mathrm{mTORC} 1$ inactivation.

\section{DISCUSSION}

Herein, we report that natural product alkyl benzoquinones are novel inhibitors of the emerging cancer drug target glutaminase, a discovery made by screening a small collection of natural products. The chemical structures of the AV and AK compounds include a fatty acid moiety, reflecting the fact that fatty acids and fatty acyl-coenzyme A derivatives can modulate glutaminase activity [9]. Thus, the possibility arises that potent endogenous modulators may exist and play significant roles in regulating glutaminase activity under various physiological conditions.

GLS2 expression was reported to be significantly increased in 186 human colon carcinoma samples. GLS2 depletion in HT1299 lung cancer cells by siRNA increased the apoptosis cell population and, in combination with the inducers of ROS-dependent apoptosis doxorubicin or n-butyrate, further augmented the percentage of apoptotic cells [12]. These findings suggest a potential role of GLS2 in anti-cancer therapies. In our study, the relative inhibitory activities of the alkyl benzoquinones of glutaminase were generally consistent with their respective activities for anti-cancer cell growth (Table1, Table S1, and Figure 3B). Moreover, the GLS2 depletion in HepG2 cells also diminished cell growth (Figure 3D). Furthermore, AV-1 and GLS2 depletion significantly suppressed the colony formation of HepG2 (Figure 3C). Inhibition of glutaminase activity in cancer cells by alkyl benzoquinones results in a deficient nutrient supply (Figure 3E), which leads to i) an AMPK-mediated moderate ULK1 activation; ii) AMPK-mediated phosphorylations on Raptor Ser792 and Tuberin Thr1387 and a PI3K/AKT inhibition mediated dephosphorylation on Tuberin Thr1462, which results in significant mTORC1 inhibition; and ultimately iii) induction of autophagy (Figures $4 \& 5$ ). Therefore we conclude that GAB/GLS2 inhibition plays a significant role in the anti-cancer mechanisms for compound AV-1.

Glutaminase inhibitors are poorly explored, with few classes of potent allosteric inhibitors identified, e.g. BPTES and dibenzophenanthridine-968 compounds $[2-6,10]$. Co-crystallization data showed that BPTES selectively inhibits GLS1 (KGA and GAC) via binding a differentiated gating loop close to the glutamine substrate binding site and exerting an allosteric effect to lock the tetramer into a nonproductive conformation. The GAC point mutants GAC F318Y/F322S and GAC Y394L were resistant to BPTES inhibition indicating that these interacting residues, Phe 322 and Tyr 394, are important for BPTES selectivity [3]. Molecular docking studies were conducted to locate potential dibenzophenanthridine-968 binding sites and site-directed mutagenesis performed to validate the important interacting residues. For example, two key residues Arg 539 and Phe 532 were mutated to construct R539L and F532L GAC mutants; dibenzophenanthridine-968 was found to be a greatly inferior inhibitor of GAC mutants than wild type. In order to understand more about the binding site of $\mathrm{AV}$ compounds, homologous modeling, docking and mutagenesis studies were used to propose an AV-1 binding site in $\mathrm{GAB}$, and the roles of interacted residues in AV-1 selectivity for GAB over KGA. The analysis of structure-activity relationships reveals the importance of the two keto or hydroxyl group at positions 1 and 4 at the benzoquinone core and an acetate group at position 2' for AV-1 potency in inhibiting KGA and GAB. Docking studies found these side groups of AV-1 to interact with conserved residues Ser456 and Lys472 at GAB through hydrogen bonds (Figure 2A). In addition, replacement of the GAB residues Q452 and $\mathrm{K} 453$ with the corresponding KGA residues His519 and Asp520 affected glutaminase selectivity of AV-1 for GAB (GLS2) over KGA (GLS1) (Figure 2A \& 2D), presumably because these residues interact with $\mathrm{AV}-1$ through van der Waal forces. We constructed two KGA mutants corresponding to those of GAC (F318Y/F322S; Y394L) and tested their activities. As expected, BPTES was not a potent inhibitor of these two KGA mutants, whilst AV-1 inhibited both wild type KGA and the mutants to a similar degree (Figure 2D). This divergent differentiation of the isoforms of GAB and KGA presents the possible selective allosteric binding sites for GLS1 over GLS2 or GLS2 over GLS1. Moreover, these results clearly demonstrate that the proximal binding site of AV-1 was different from those of BPTES and dibenzophenanthridine- 968 .

The amino acid sequences of the glutaminase isoforms are highly homologous ( $81 \%$ identity) within their catalytic core regions (Figure S3, [37-39]). The rest of heterogeneous regions present potential differentiated allosteric pockets subjected to various modulations in enzymatic activity as evidence by inhibitors BPTES, dibenzophenanthridine-968, and AV-1. The accessibility of these allosteric pockets to small physiological molecules would allow for physiological modulations in the enzymatic activity. Thus, the possibility of identifying more allosteric pockets for modulating glutaminase isozymes merits further investigation.

\section{MATERIALS AND METHODS}

\section{Cell culture, reagents, and western blotting}

The cancer cell lines including HepG2 and A549 were obtained from American Type Culture Collection (Rockville, MD). The cells were cultured in DMEM 
medium with $10 \% \mathrm{FBS}(\mathrm{v} / \mathrm{v})$ and penicillin (100 units/ $\mathrm{ml}) /$ streptomycin $(100 \mathrm{mg} / \mathrm{ml})$. Cultures were maintained in a humidified incubator at $37^{\circ} \mathrm{C}$ in $5 \% \mathrm{CO}_{2} / 95 \%$ air. Dulbecco's Modified Eagle Medium (DMEM), fetal bovine serum (FBS), penicillin, streptomycin, and all other tissue culture reagents were obtained from GIBCO/BRL Life Technologies (Grand Island, NY). Antibodies to GAPDH, caspase 3, LC3B, p-ULK1(S317), p-ULK1(S757), Beclin1, p-BCL2(S70), p70S6K, p-p70S6K(T389), 4E-BP1, $\alpha$-tubulin, 4E-BP, p-4EBP(S65), p-eIF4B(S422), mTOR, p-mTOR(S2481), p-mTOR(S2448), p-Raptor(S792), Tuberin, p-Tuberin(T1462), p-Tuberin(T1387), p-AMPK(T172) and p-AMPK(S108) were from Cell Signaling Technology; antibody to p-Beclin1(S15) was from Abbiotec; antibodies to KGA and Raptor were from Abcam; antibodies to BCL2 were from Upstate Biotechnology; antibodies to $\alpha$-tubulin were from Chemicon International; antibodies to ULK1, $\beta$-tublin and AMPK were from GeneTex; antibodies to GAB were from abnova and Gene Tex; antibodies to GAC were from ProteinTech Group; antibodies to p-mTOR(S2446) and AMPK were from Millipore; anti-mouse and anti-rabbit IgGs were obtained from PerkinElmer. Alkyl benzoquinones were purified from A. virens and A. kusukuensis [27, 28]. Compound C was obtained from Sigma-Aldrich (St. Louis, MO). Western blotting was performed as described [40].

\section{Preparation of BPTES}

The synthesis of BPTES was modified from one previously published [41]. Step 1 Procedure: A mixture of thiosemicarbazide (1.82 $\mathrm{g}, 20 \mathrm{mmol})$, thiodipropionic acid $(1.78 \mathrm{~g}, 10 \mathrm{mmol})$ and $\mathrm{POCl}_{3}(15 \mathrm{ml})$ was heated at $90{ }^{\circ} \mathrm{C}$ for $3 \mathrm{~h}$. After obtaining a clear solution, the reaction mixture was cooled and poured in to $100 \mathrm{~g}$ of ice. The solid was filtered and the filtrate was adjusted to $\mathrm{pH} 14$ using $5 \mathrm{~N} \mathrm{NaOH}$. The resulting white solid was filtered, washed with water and dried to give bis(aminothioldiazole) (2.3 g, 80\%).

Step 2 Procedure: $\mathrm{NaH}(60 \%, 660 \mathrm{mg}, 16.5 \mathrm{mmol})$ was added to a solution of bis-(aminothioldiazole) (1.586 $\mathrm{g}, 5.5 \mathrm{mmol})$ in THF $(200 \mathrm{~mL})$ at $0^{\circ} \mathrm{C}$. After being stirred for $5 \mathrm{~min}$, phenylacetyl chloride $(2.2 \mathrm{~mL}, 16.5 \mathrm{mmol})$ was added to the reaction mixture and the mixture was continuously stirred at room temperature for $16 \mathrm{~h}$. Water was added to quench the reaction and the resulting mixture was extracted with ethyl acetate. The organic layers were washed with brine, dried over sodium sulfate, filtered, and concentrated in vacuo to furnish the crude BPTES. The solid was redissolved in DMSO and triturated with methanol to give BPTES as a white solid (518 mg, 18\%) m.p 234-235 ${ }^{\circ} \mathrm{C}$; ${ }^{1} \mathrm{H}-\mathrm{NMR}\left(300 \mathrm{MHz},\left(\mathrm{CD}_{3}\right)_{2} \mathrm{SO}\right): 2.92$ (4H, t, $J=7.2 \mathrm{~Hz}), 3.24$ (4H, t, $J=7.2 \mathrm{~Hz}), 7.31$ (10H, m), 12.67 (2H, NH). Purity: 99.12\% (RS-HPLC)

\section{Cloning, expression, and purification of glutaminases (KGA and GAB)}

The cDNA clone MGC:33744 IMAGE:5263220 (Invitrogen Inc., USA) was used to amplify a KGA cDNA fragment of 1644 bp (residues Leu123-Leu669) by PCR with primers 5'-ATACGCGGATCCCTGGTGGCC TCAGGTGAAAA-3' and 5'-GTAAAGGAAAAAA GCGGCCGCTTACAACAATCCATCAAGAT-3'

containing BamHI and NotI, respectively, for subsequent sub-cloning. The cDNA fragment of KGA digested with BamHI and NotI at each end was ligated into the pET28a(+) vector (Novagen) and expressed as a his-tag fusion. Escherichia coli strain BL21(DE3)pLysS was transformed with the resulting plasmid, pET28a(+)-KGA, and then cultured in Luria-Bertani broth medium in the presence of kanamycin. Once the cultures reached an absorbance at $600 \mathrm{~nm}$ of 0.5 to 0.6 , they were induced with $0.5 \mathrm{mM}$ isopropyl $\beta$-D-1-thiogalactopyranoside (IPTG) overnight. The cells were harvested by centrifugation at $10,000 \mathrm{rpm}$ for $15 \mathrm{~min}$ at $4^{\circ} \mathrm{C}$ and resuspended in lysis buffer (5 mM Tris-HCl [pH 8.0], 50 mM NaH${ }_{2} \mathrm{PO}_{4}, 300$ $\mathrm{mM} \mathrm{NaCl}$ and $10 \mathrm{mM}$ imidazole) for sonication. The filtered supernatant was applied to an affinity column, HisTrapTM HP 1 x 5 mL (GE Healthcare), and washed with lysis buffer containing $10 \mathrm{mM} \beta$-mercaptoethanol $(\beta-\mathrm{ME}), 5 \%$ glycerol, $0.1 \%$ Triton $\mathrm{X}-100$ and $100-150$ $\mathrm{mM}$ imidazole. His-tagged KGA was eluted with lysis buffer containing $10 \mathrm{mM} \beta-\mathrm{ME}, 5 \%$ glycerol, $0.1 \%$ Triton $\mathrm{X}-100$ and $500 \mathrm{mM}$ imidazole. The eluted protein was exchanged to $20 \mathrm{mM}$ Tris- $\mathrm{HCl}$ ( $\mathrm{pH} \mathrm{8.0),} 300 \mathrm{mM} \mathrm{NaCl}$, $10 \%$ glycerol, and $2 \mathrm{mM}$ dithiothreitol by using a PD-10 Desalting column (GE Healthcare). The concentration of the purified protein was determined by use of the Bio-Rad protein assay reagent with a standard curve plotted against bovine serum albumin.

Equivalent construct of GAB (Pro56-Val602) was amplified from the cDNA clone MGC: 195512 IMAGE: 100066375 (Invitrogen Inc., USA). The sequences of the primers were: 5'- GGAGGCGCGGATCCCCGC AGCACCAGGATCATG-3' (BamHI) and 5' - GCAAGGAAAAAAGCGGCCGCAGCG CTATACCATGCTTTCTAAGTTCTC-3' (Notl). The resultant GAB-expression vector, pET28a(+)-GAB, was transformed into Escherichia coli strain C41(DE3)pLysS (Yeastern Biotech Co., Taiwan). The expression protocol was followed as that protocol of KGA construct. The cells after induction were collected and resuspended in lysis buffer (50 mM Tris- $\mathrm{HCl}$ pH 8.5, $500 \mathrm{mM} \mathrm{NaCl,} \mathrm{10 \%}$ glycerol, and $10 \mathrm{mM} \beta-\mathrm{ME}$ ) for sonication. The filtered supernatant was applied to a $\mathrm{Ni}^{+}$-charged affinity column (GE Healthcare), and washed with lysis buffer containing $50 \mathrm{mM}$ imidazole. His-tagged GAB was eluted with lysis buffer containing $200 \mathrm{mM}$ imidazole. The eluted GAB was exchanged to $20 \mathrm{mM}$ Tris- $\mathrm{HCl}$ ( $\mathrm{pH} 8.0$ ), $300 \mathrm{mM}$ $\mathrm{NaCl}, 50 \%$ glycerol, and $2 \mathrm{mM}$ dithiothreitol by using a 
PD-10 Desalting column (GE Healthcare).

\section{Glutaminase activity assays and kinetic studies}

Human glutaminase activity was measured using a two-step assay as described previously [42, 43]. A microtiter assay format was used to facilitate the collection of multiple data points. Typically, $10 \mu \mathrm{l}$ of compound, which was dissolved in DMSO, was added to $80 \mu \mathrm{l}$ of an initial assay mix (0.1 mM hKGA, $0.2 \mathrm{mM}$ EDTA and $50 \mathrm{mM}$ Tris/acetate, $\mathrm{pH}$ 8.6). Samples were incubated at $25^{\circ} \mathrm{C}$ for $10 \mathrm{~min}$ and then $10 \mu \mathrm{l}$ of $200 \mathrm{mM}$ glutamine were added to the reaction mix. After adding glutamine, the reactions were incubated at $37^{\circ} \mathrm{C}$ for $60 \mathrm{~min}$ and then quenched by the addition of $10 \mu \mathrm{l}$ of $0.6 \mathrm{M} \mathrm{HCl}$. Subsequently, $100 \mu$ of a second reaction mixture (3.7 units of purified bovine liver glutamate dehydrogenase, $160 \mathrm{mM}$ Tris/acetate [pH 9.4], $400 \mathrm{mM}$ hydrazine, 5 $\mathrm{mM} \mathrm{ADP}$ and $2 \mathrm{mM} \mathrm{NAD}^{+}$) was added to the stopped first reaction mixture and incubated for $30 \mathrm{~min}$ at $25^{\circ} \mathrm{C}$. The absorbance at $340 \mathrm{~nm}$ was measured using a Wallac Victor2V plate reader (PerkinElmer), fitted with a $340 \mathrm{~nm}$ excitation filter. Sample absorbance was measured against a blank, containing no human glutaminase.

The kinetic constants $\mathrm{K}_{\mathrm{m}}$ and $\mathrm{V}_{\max }$ were determined by using Kaleida Graph software (Synergy Software) to calculate a non-linear least-squares fit for all the data points to the Michaelis-Menten equation. The Ki values for $\mathrm{AV}-1$ were determined by using the ChengPrusoff equation; $\mathrm{Ki}=\mathrm{IC}_{50} /\left(1+[\mathrm{S}] / \mathrm{K}_{\mathrm{m}}\right)$ where $[\mathrm{S}]$ is the concentration of the glutamine.

\section{In vitro carcinoma cell growth inhibition and colony formation assays}

A549 lung carcinoma and HepG2 hepatocarcinoma cells were seeded at 4000 and 10000 cells/well respectively in 96-well plates, as previously described for cell growth inhibition assay $[27,44]$. To assess anchoragedependent colony formation effect, the cells (1000 cells/ well) were seeded in a six-well plate for 20-24 h prior to treatments of vehicle DMSO or compounds as indicated. Culture medium containing vehicle DMSO or compounds as indicated was changed twice a week. After a 21-day treatment, the cell colonies were rinsed with PBS, fixed in $3: 1$ ratio of methanol and acetic acid, stained with $0.05 \%$ crystal violet.

\section{Determination of intracellular glutaminase activity and glutamate levels}

Determination of intracellular glutaminase activity was modified from above described two-step glutaminase assay. Step one mixture of a $100 \mu \mathrm{l}$ solution containing
$20 \mu \mathrm{g}$ cell lysate, $0.2 \mathrm{mM}$ EDTA, $50 \mathrm{mM}$ Tris/acetate $(\mathrm{pH}$ 8.6), and $150 \mathrm{mM}$ phosphate, was incubated at $37^{\circ} \mathrm{C}$ for 60 min and the reaction was stopped by $10 \mu \mathrm{l}$ of $0.6 \mathrm{M} \mathrm{HCl}$. Subsequently, $30 \mu \mathrm{l}$ of the stopped reaction was subjected to the second reaction as described above for glutaminase assay. Glutamate levels were measured by using the Amplex Red Glutamine Acid/Glutamate oxidase assay kit (Invitrogen) according to manufacturer's instructions.

\section{Mutagenesis and docking}

Site-directed mutagenesis was performed on the pET28a(+)-GAB or pET28a(+)-KGA plasmids using PfuUltra II Fusion HS DNA polymerase (Agilent Technologies) to create the GAB and KGA mutants. The deep view program (Version 3.7) of the Swiss-MODEL server was used to generate the three dimensional structure of homologous modeled GAB. Using the integrated sequence alignment tools and structural superimposition algorithms, the target sequence was mapped on the modeling templates. The sequence alignment was compared with crystal structure of the GAC (PDB code: 3UO9) and the resulting model of GAB was analyzed using the Swiss-PDB server. Threading and folding recognition of GAB model was developed by PHYRE2 web server. Docking studies were performed with AutoDock Vina. Autodock input files were prepared with MGLTools 1.5.6. Molecules were drawn in ChemBioOffice 2010, and energy minimized using the MM2 force field in Chemdraw 3D. Visualization was performed with PyMOL 1.3, and graphics were prepared in that software. SWISS MODEL: http://swissmodel. expasy.org/ and PHYRE2: http:/www.sbg.bio.ic.ac.uk/ phyre $2 /$ html/page.cgi?id=index.

\section{GLS2 gene silencing}

The knock down of GLS2 was carried out using two distinct shRNA plasmids (clone ID: TRCN0000051323 and TRCN0000051327, Academia Sinica, Taiwan) directed against the two isoforms of GLS2 enzyme (GAB and LGA). The shRNA plasmid (TRCN0000231759, Academia Sinica, Taiwan) against of GFP was as a negative control. The shRNA plasmids were transfected in A549 cells, or HepG2 cells using FuGene $6^{\mathrm{TM}}$ (Roche). Stable cell clones were grown in the presence of $1 \mu \mathrm{g} / \mathrm{mL}$ puromycin. Established cell lines were further verified by anti-GAB western blot analysis to assess the cells growth.

\section{Funding}

This work was funded by the Ministry of Economic Affairs, R.O.C. "101-EC-17-A-02-04-1099”, "102-EC17-A-02-04-1099”, “103-EC-17-A-22-1099”, the National 
Science Council of Taiwan (NSC 102-2628-B-400-002MY3), and the National Health Research Institutes, Taiwan, R.O.C.

\section{ACKNOWLEDGMENTS}

We would like to thank the Academia Sinica siRNA Core Facility for the shRNA used in this study, and Dr. Chung-I Chang at the Academia Sinica, Taiwan, for encouraging the molecular docking studies. Ya-Qi Qiu is currently a $\mathrm{PhD}$ student in the Graduate Program of Biotechnology in Medicine, which is sponsored by the National Health Research Institutes and National Tsing Hua University.

\section{Author Contributions}

Y.Z.L. conducted molecular docking experiments. Y.Z.L., C.W.Y., H.Y.C., H.Y.H., and Y.Q.Q. performed the cell biology and molecular biology experiments. I.S.C and H.S.C. contributed to the collection of $\mathrm{AV}$ and $\mathrm{AK}$ series of compounds. J.C.L. and C.R. K. designed and synthesized the BPTES. Y.S.C and C.H.L advised with the concept. Y.Z.L., C.W.Y, C.H.L, and S.J.L. interpreted the data and wrote the manuscript. S.J.L. supervised the experimental design, the interpretation of the data, and the composition of the manuscript.

\section{Competing Financial Interests Statement}

The authors declare no competing financial interests.

\section{REFERENCES}

1. Erickson JW and Cerione RA. Glutaminase: a hot spot for regulation of cancer cell metabolism? Oncotarget. 2010; 1(8):734-740.

2. Thangavelu K, Pan CQ, Karlberg T, Balaji G, Uttamchandani M, Suresh V, Schuler H, Low BC and Sivaraman J. Structural basis for the allosteric inhibitory mechanism of human kidney-type glutaminase (KGA) and its regulation by Raf-Mek-Erk signaling in cancer cell metabolism. Proceedings of the National Academy of Sciences of the United States of America. 2012; 109(20):7705-7710.

3. DeLaBarre B, Gross S, Fang C, Gao Y, Jha A, Jiang F, Song JJ, Wei W and Hurov JB. Full-length human glutaminase in complex with an allosteric inhibitor. Biochemistry. 2011; 50(50):10764-10770.

4. Katt WP, Ramachandran S, Erickson JW and Cerione RA. Dibenzophenanthridines as inhibitors of glutaminase $\mathrm{C}$ and cancer cell proliferation. Molecular cancer therapeutics. 2012; 11(6):1269-1278.

5. Le A, Lane AN, Hamaker M, Bose S, Gouw A, Barbi J,
Tsukamoto T, Rojas CJ, Slusher BS, Zhang H, Zimmerman LJ, Liebler DC, Slebos RJ, Lorkiewicz PK, Higashi RM, Fan TW, et al. Glucose-independent glutamine metabolism via TCA cycling for proliferation and survival in B cells. Cell metabolism. 2012; 15(1):110-121.

6. Shukla K, Ferraris DV, Thomas AG, Stathis M, Duvall B, Delahanty G, Alt J, Rais R, Rojas C, Gao P, Xiang Y, Dang $\mathrm{CV}$, Slusher BS and Tsukamoto T. Design, synthesis, and pharmacological evaluation of bis-2-(5-phenylacetamido1,2,4-thiadiazol-2-yl)ethyl sulfide (BPTES) analogs as glutaminase inhibitors. Journal of medicinal chemistry. 2012; 55(23):10551-10563.

7. Mates JM, Segura JA, Martin-Rufian M, Campos-Sandoval JA, Alonso FJ and Marquez J. Glutaminase isoenzymes as key regulators in metabolic and oxidative stress against cancer. Current molecular medicine. 2013; 13(4):514-534.

8. Cassago A, Ferreira AP, Ferreira IM, Fornezari C, Gomes ER, Greene KS, Pereira HM, Garratt RC, Dias SM and Ambrosio AL. Mitochondrial localization and structurebased phosphate activation mechanism of Glutaminase C with implications for cancer metabolism. Proceedings of the National Academy of Sciences of the United States of America. 2012; 109(4):1092-1097.

9. Kvamme E and Torgner IA. Regulatory effects of fatty acyl-coenzyme A derivatives on phosphate-activated pig brain and kidney glutaminase in vitro. The Biochemical journal. 1975; 149(1):83-91.

10. Wang JB, Erickson JW, Fuji R, Ramachandran S, Gao P, Dinavahi R, Wilson KF, Ambrosio AL, Dias SM, Dang $\mathrm{CV}$ and Cerione RA. Targeting mitochondrial glutaminase activity inhibits oncogenic transformation. Cancer cell. 2010; 18(3):207-219.

11. Hu W, Zhang C, Wu R, Sun Y, Levine A and Feng Z. Glutaminase 2, a novel p53 target gene regulating energy metabolism and antioxidant function. Proceedings of the National Academy of Sciences of the United States of America. 2010; 107(16):7455-7460.

12. Giacobbe A, Bongiorno-Borbone L, Bernassola F, Terrinoni A, Markert EK, Levine AJ, Feng Z, Agostini M, Zolla L, Agro AF, Notterman DA, Melino G and Peschiaroli A. p63 regulates glutaminase 2 expression. Cell cycle 2013; 12(9):1395-1405.

13. Velletri T, Romeo F, Tucci P, Peschiaroli A, AnnicchiaricoPetruzzelli M, Niklison-Chirou MV, Amelio I, Knight RA, Mak TW, Melino G and Agostini M. GLS2 is transcriptionally regulated by $\mathrm{p} 73$ and contributes to neuronal differentiation. Cell cycle 2013; 12(22):35643573.

14. Szeliga M, Zgrzywa A, Obara-Michlewska $M$ and Albrecht J. Transfection of a human glioblastoma cell line with liver-type glutaminase (LGA) down-regulates the expression of DNA-repair gene MGMT and sensitizes the cells to alkylating agents. Journal of neurochemistry. 2012; 123(3):428-436.

15. Xiang L, Xie G, Liu C, Zhou J, Chen J, Yu S, Li J, Pang 
$\mathrm{X}$, Shi $\mathrm{H}$ and Liang $\mathrm{H}$. Knock-down of glutaminase 2 expression decreases glutathione, $\mathrm{NADH}$, and sensitizes cervical cancer to ionizing radiation. Biochimica et biophysica acta. 2013; 1833(12):2996-3005.

16. Kim J, Kundu M, Viollet B and Guan KL. AMPK and mTOR regulate autophagy through direct phosphorylation of Ulk1. Nature cell biology. 2011; 13(2):132-141.

17. Young MM, Kester $M$ and Wang HG. Sphingolipids: regulators of crosstalk between apoptosis and autophagy. Journal of lipid research. 2013; 54(1):5-19.

18. Leontieva OV and Blagosklonny MV. M(o)TOR of pseudohypoxic state in aging: rapamycin to the rescue. Cell cycle 2014; 13(4):509-515.

19. Leontieva OV and Blagosklonny MV. Yeast-like chronological senescence in mammalian cells: phenomenon, mechanism and pharmacological suppression. Aging. 2011; 3(11):1078-1091.

20. Duran RV and Hall MN. Glutaminolysis feeds mTORC1. Cell cycle 2012; 11(22):4107-4108.

21. Roach PJ. AMPK -> ULK1 -> autophagy. Molecular and cellular biology. 2011; 31(15):3082-3084.

22. Kang R, Zeh HJ, Lotze MT and Tang D. The Beclin 1 network regulates autophagy and apoptosis. Cell death and differentiation. 2011; 18(4):571-580.

23. Russell RC, Tian Y, Yuan H, Park HW, Chang YY, Kim J, Kim H, Neufeld TP, Dillin A and Guan KL. ULK1 induces autophagy by phosphorylating Beclin-1 and activating VPS34 lipid kinase. Nature cell biology. 2013; 15(7):741750.

24. Harvey AL. Natural products in drug discovery. Drug discovery today. 2008; 13(19-20):894-901.

25. Newman DJ and Cragg GM. Natural products as sources of new drugs over the 30 years from 1981 to 2010. Journal of natural products. $2012 ; 75(3): 311-335$.

26. Snodgrass PJ and Lund P. Allosteric properties of phosphate-activated glutaminase of human liver mitochondria. Biochimica et biophysica acta. 1984; 798(1):21-27.

27. Chang HS, Lin YJ, Lee SJ, Yang CW, Lin WY, Tsai IL and Chen IS. Cytotoxic alkyl benzoquinones and alkyl phenols from Ardisia virens. Phytochemistry. 2009; 70(1718):2064-2071.

28. Su TJ, Chang HS, Peng CF, Lee SJ and Chen IS. Antitubercular Resorcinols and Cytotoxic Alkyl Benzoquinones from Ardisia kusukuensis. Taiwan Pharmaceutical Journal 2009; 61:89-105.

29. Ouyang L, Shi Z, Zhao S, Wang FT, Zhou TT, Liu B and Bao JK. Programmed cell death pathways in cancer: a review of apoptosis, autophagy and programmed necrosis. Cell Proliferat. 2012; 45(6):487-498.

30. Kabeya Y, Mizushima N, Yamamoto A, Oshitani-Okamoto S, Ohsumi Y and Yoshimori T. LC3, GABARAP and GATE16 localize to autophagosomal membrane depending on form-II formation. Journal of cell science. 2004; 117(Pt
13):2805-2812.

31. Allan LA and Clarke PR. Apoptosis and autophagy: Regulation of caspase-9 by phosphorylation. The FEBS journal. 2009; 276(21):6063-6073.

32. Peterson RT, Beal PA, Comb MJ and Schreiber SL. FKBP12-rapamycin-associated protein (FRAP) autophosphorylates at serine 2481 under translationally repressive conditions. The Journal of biological chemistry. 2000; 275(10):7416-7423.

33. Huang $\mathrm{S}$ and Houghton PJ. Targeting mTOR signaling for cancer therapy. Current opinion in pharmacology. 2003; 3(4):371-377.

34. Cheng SW, Fryer LG, Carling D and Shepherd PR. Thr2446 is a novel mammalian target of rapamycin (mTOR) phosphorylation site regulated by nutrient status. The Journal of biological chemistry. 2004; 279(16):1571915722.

35. Manning BD, Tee AR, Logsdon MN, Blenis J and Cantley LC. Identification of the tuberous sclerosis complex-2 tumor suppressor gene product tuberin as a target of the phosphoinositide 3-kinase/akt pathway. Molecular cell. 2002; 10(1):151-162.

36. Inoki $\mathrm{K}, \mathrm{Zhu} \mathrm{T}$ and Guan KL. TSC2 mediates cellular energy response to control cell growth and survival. Cell. 2003; 115(5):577-590.

37. Elgadi KM, Meguid RA, Qian M, Souba WW and Abcouwer SF. Cloning and analysis of unique human glutaminase isoforms generated by tissue-specific alternative splicing. Physiological genomics. 1999; 1(2):5162.

38. Gomez-Fabre PM, Aledo JC, Del Castillo-Olivares A, Alonso FJ, Nunez De Castro I, Campos JA and Marquez J. Molecular cloning, sequencing and expression studies of the human breast cancer cell glutaminase. The Biochemical journal. 2000; 345 Pt 2:365-375.

39. Holcomb T, Taylor L, Trohkimoinen J and Curthoys NP. Isolation, characterization and expression of a human brain mitochondrial glutaminase cDNA. Brain research Molecular brain research. 2000; 76(1):56-63.

40. Yang CW, Yang YN, Liang PH, Chen CM, Chen WL, Chang HY, Chao YS and Lee SJ. Novel smallmolecule inhibitors of transmissible gastroenteritis virus. Antimicrobial agents and chemotherapy. 2007; 51(11):3924-3931.

41. Newcomb RW. (2002). United States Patent No. 6,451, 828 B1. Selective Inhibition of Glutaminase by Bis thiadiazoles.

42. Kenny J, Bao Y, Hamm B, Taylor L, Toth A, Wagers $\mathrm{B}$ and Curthoys NP. Bacterial expression, purification, and characterization of rat kidney-type mitochondrial glutaminase. Protein expression and purification. 2003; 31(1):140-148.

43. Robinson MM, McBryant SJ, Tsukamoto T, Rojas C, Ferraris DV, Hamilton SK, Hansen JC and Curthoys 
NP. Novel mechanism of inhibition of rat kidney-type glutaminase by bis-2-(5-phenylacetamido-1,2,4-thiadiazol2-yl)ethyl sulfide (BPTES). The Biochemical journal. 2007; 406(3):407-414.

44. Wu CM, Yang CW, Lee YZ, Chuang TH, Wu PL, Chao YS and Lee SJ. Tylophorine arrests carcinoma cells at G1 phase by downregulating cyclin A2 expression. Biochemical and biophysical research communications. 2009; 386(1):140145. 